\title{
Breast cancer and synchronous multiple primary lung adenocarcinomas with heterogeneous mutations: a case report
}

\author{
Bo Jin ${ }^{1 *}$, Simeng Zhang ${ }^{1}$, Xin Chuang ${ }^{1}$, Ping Yu¹, Ying Chen ${ }^{1}$, Yuee Teng ${ }^{1}$, Xiaofang Che ${ }^{1}$, Yibo Fan', \\ Chunlei Zheng ${ }^{1}$, Xiaohan $\mathrm{Li}^{3}$, Xueshan Qiu' ${ }^{2}$ Xiujuan Qu' and Yunpeng Liu ${ }^{1}$
}

\begin{abstract}
Background: Multiple primary malignant tumors (MPMT) refers to the presence of two or more primary cancers of different organs in the same patient. MPMT is a sparse disease in the past, but there has been a gradual increase in the morbidity. Since multiple primary malignant tumors treatment methods differ, it is essential for clinicians to be able to distinguish between separate primary lesions and metastasis.

Case presentation: We present the case of a 57-year-old woman with MPMT presenting with cancer in the left breast and synchronous double primary lung adenocarcinomas. We used IHC and epidermal growth factor receptor(EGFR)mutation to analyze genomic alteration profiles in the patient to validate the difference among the pathological assessments and the clinical differences between double primary lesions of lung and breast. EGFR gene analysis of breast cancer lesion revealed no mutations. The left and right lower lobe lung adenocarcinomas contained EGFR gene mutations: an L858R point mutation in exon 21 in the left lesion and a deletion mutation in exon 19 in the right lesion. The breast cancer and both lung adenocarcinomas were surgically resected. To date, the patient has remained disease-free.

Conclusions: Both pathological and molecular assessment adapted in the current study appeared necessary. Mutational analysis of the EGFR gene provided important information not only in the diagnosis and but also in the treatment of MPMT.
\end{abstract}

Keywords: Multiple primary malignant tumors, EGFR mutation, Multiple primary lung adenocarcinomas, Synchronous

\section{Background}

Multiple primary malignant tumors (MPMT) refers to the presence of two or more primary cancers of different origins in the same patient. This uncommon condition is classified as synchronous or metachronous depending on the time of diagnosis: synchronous when the time between diagnosis of the first and second primary tumors is less than 6 months and metachronous when that period is more than 6 months [1]. The reported incidence of MPMT in cancer patients ranges from 0.73 to $11.7 \%$ among cancer patients [2, 3].

\footnotetext{
* Correspondence: jb_cmu@hotmail.com

'Department of Medical Oncology, The First Hospital, China Medical University, Shenyang, China

Full list of author information is available at the end of the article
}

Here, we present a case of MPMT which have breast cancer and lung adenocarcinoma with heterogeneous epidermal growth factor receptor (EGFR) mutation status.

\section{Case presentation}

In February 2016, a 57-year-old woman was admitted to our hospital for evaluation of a breast mass and multiple pulmonary nodules. $\mathrm{AF}^{18}$-fluorodeoxyglucose (FDG) positron emission tomography computed tomography (PET-CT) scan performed at the Shengjing Hospital of China Medical University showed a left breast mass with a FDG maximal standardized uptake value (SUVmax) of 4.23 (Fig. 1a), a left lower lung lobe (LLL) nodule measuring about $1.1 \mathrm{~cm}$ in diameter with increased FDG uptake (SUVmax = 2.79; Fig. $1 \mathrm{~b})$, and a right lower lung 

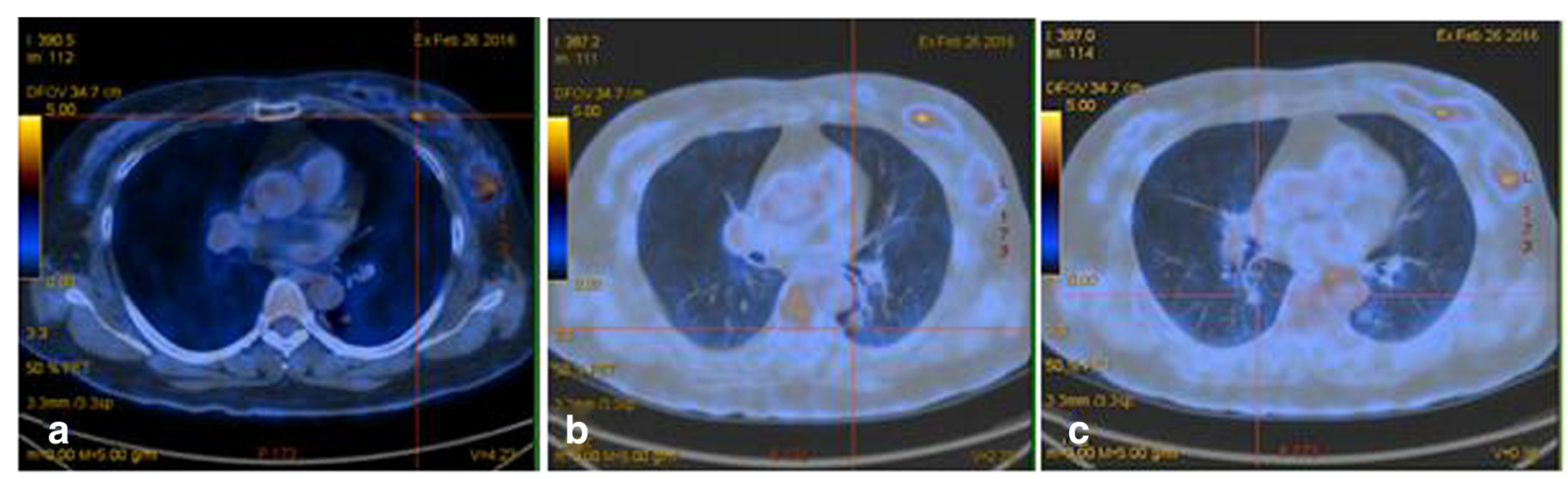

Fig. 1 Positron Emission Tomography-computed tomography. The primary lesion in the left breast (a); The primary lesion in the left lower lobe of the lung (b) and the lesion in the right lower lobe of the lung (c)

lobe (RLL) nodule measuring about $0.8 \mathrm{~cm}$ with normal FDG uptake (Fig. 1c). The LLL lesion was considered malignant, whereas the RLL lesion was not diagnosed as benign or malignant. Sequential surgery for resection of the breast cancer and LLL lesion was considered a reasonable course of action.

A left radical mastectomy was performed on March 2nd, 2016. Postoperative pathology showed ductal carcinoma in situ (high grade). Immunohistochemical (IHC) staining indicated that the lesion was estrogen receptor(ER) negative (Fig. 2a), progesterone receptor(PR) negative (Fig. 2b), C-erbB-2 positive carcinoma in situ $(3+$; Fig. 2c) and thyroid transcription factor-1(TTF-1) negative(Fig. 2d). The margins were negative. Sentinel lymph node analysis revealed reactive hyperplasia in the axillary lymph node $(0 / 5,0 / 10)$. The pathological stage was pTisN0M0, 0 stage according to AJCC version 7.0 [4]. EGFR gene analysis (Fig. 3) revealed no mutations.

A left lower lobectomy with lymph node dissection was performed at our hospital in April 2016. Postoperative pathology identified a highly to moderately differentiated adenocarcinoma (gland bubble type, 90\%; lepidic
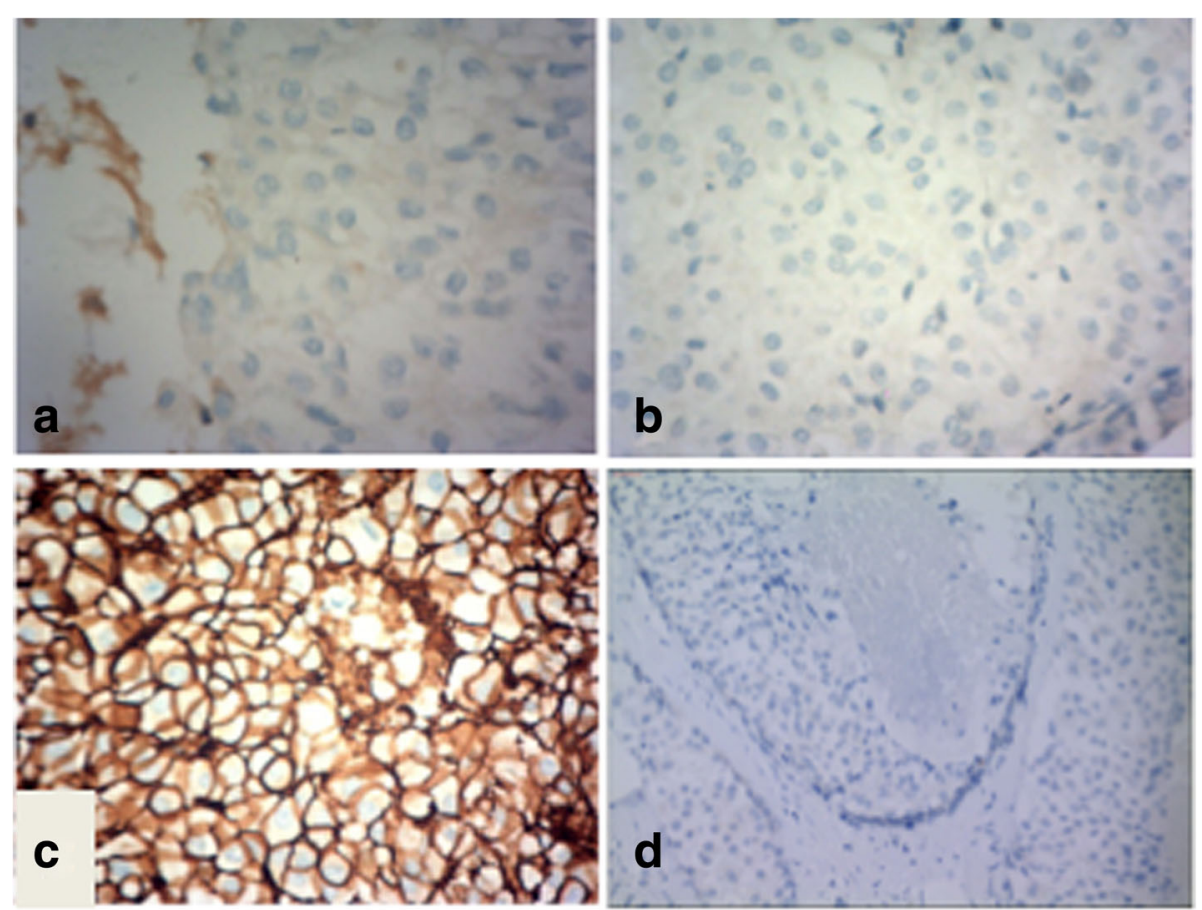

Fig. 2 Immunohistopathological analysis of the postoperative breast lesion demonstrated negative staining for ER (a), PR (b) and TTF-1 (d), but positive staining for C-erbB-2 (c) 


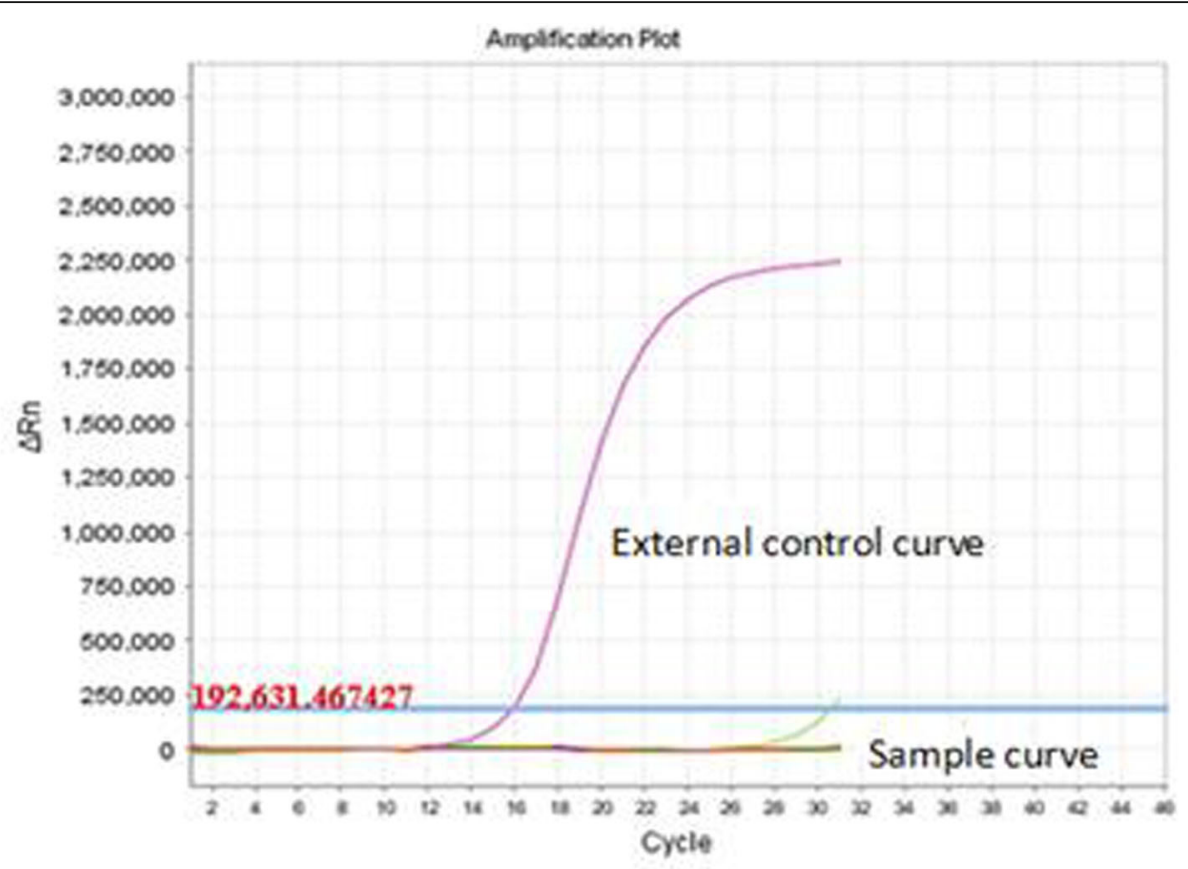

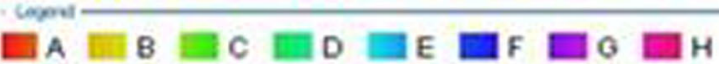

Fig. 3 EGFR gene analysis of the left breast lesion revealed no mutations

growth pattern, 10\%). Cancer cells were not detected in the lymph nodes. By IHC analysis, the lesion was CK7 positive (Fig. 4a), P63 negative, napsin A positive (Fig. 4b), TTF-1 positive (Fig. 4c), ALK D5F3 negative, ALK negative, and Ki-67 positive (5\%). EGFR gene analysis was performed in June 2016 and showed an L858R mutation in exon 21 (Fig. 5). The pathological results of breast cancer (pTisN0M0,stage 0) and lung cancer (pT1aNOM0, stage IA according to AJCC version 7.0 [5]) showed that the patient has MPMT.

A chest CT scan performed 2 months after the left lower lobectomy showed that the small ground glass nodule in the RLL had increased in size compared with the scan performed 5 months earlier. Since the LLL lesion expressed mutated EGFR, the patient was prescribed gefitinib $250 \mathrm{mg}$ daily starting in August 2016. A follow-up chest CT scan performed 40 days later showed a stable RLL nodule.

A right lower lobectomy with lymph node dissection was performed in our hospital in September 2016. Postoperative pathology identified an adenocarcinoma (alveolar type, $40 \%$; growing along the alveolar wall, $60 \%$ ). There was no lymph node metastasis and the surgical margins were clear. By IHC analysis, the lesion was CK A1 positive (Fig. 6a), CK5/6 negative, CK7 positive (Fig. 6b), P63 negative, $\mathrm{P} 40$ negative, napsin A positive (Fig. 6c), TTF-1 positive (Fig. 6d), CD56 negative, synaptophysin negative, and Ki-67 positive (10\%). Analysis of the EGFR gene showed a

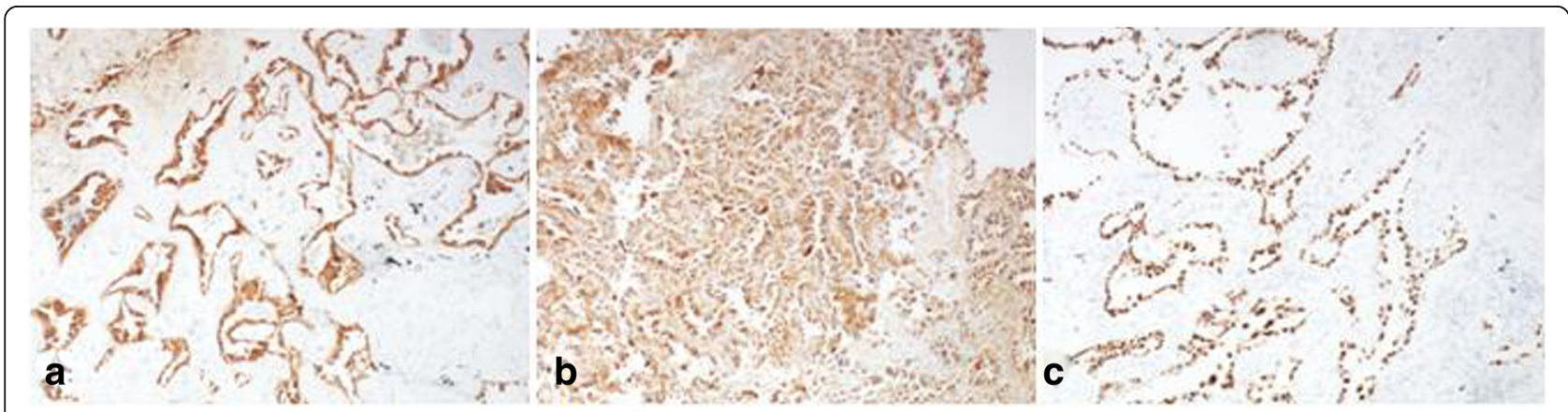

Fig. 4 Immunohistopathological analysis of the postoperative left lung lesion demonstrated positive staining for CK7 (a), napsin A (b), and TTF-1 (c) 


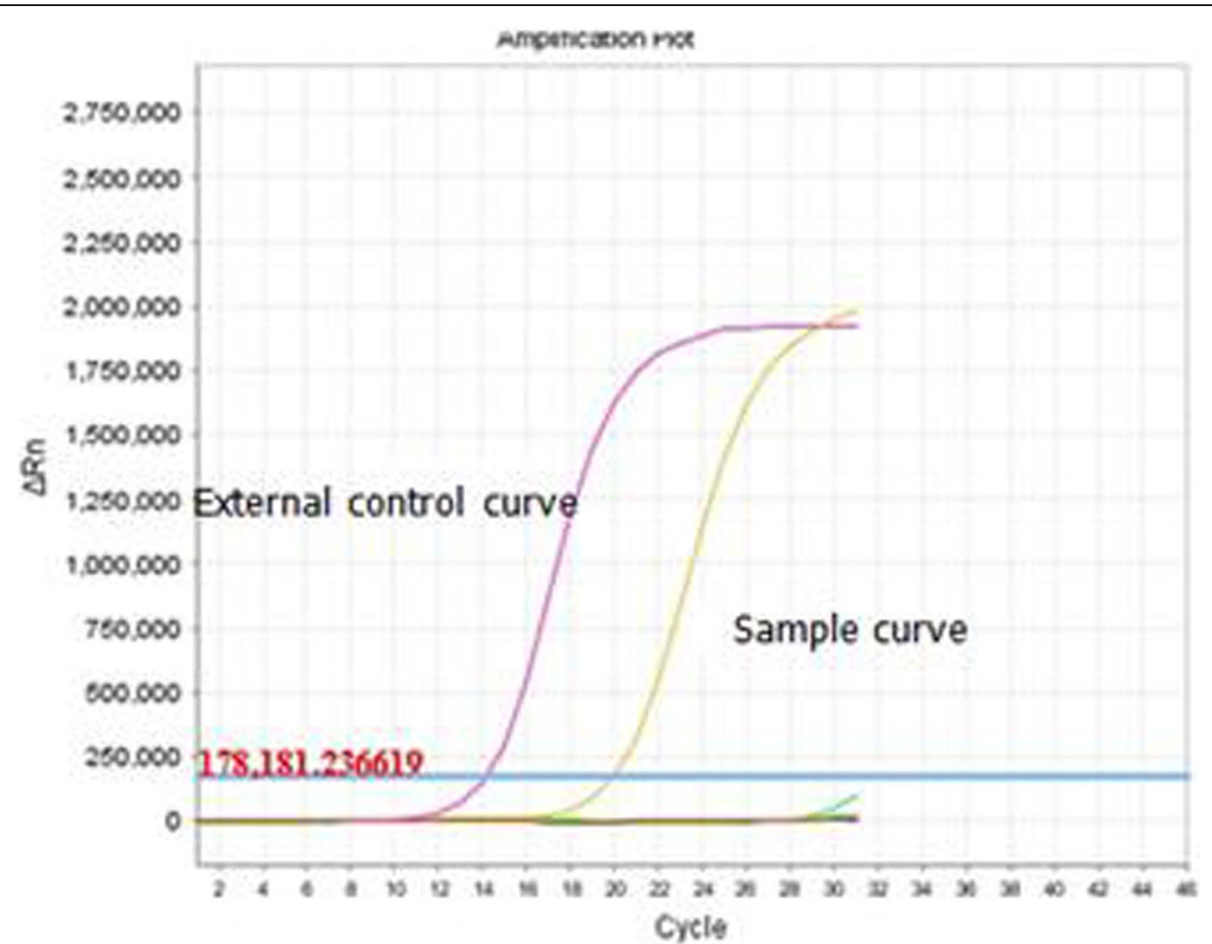

Fig. 5 EGFR gene analysis of the left lung lesion revealed an L858R mutation in exon 21

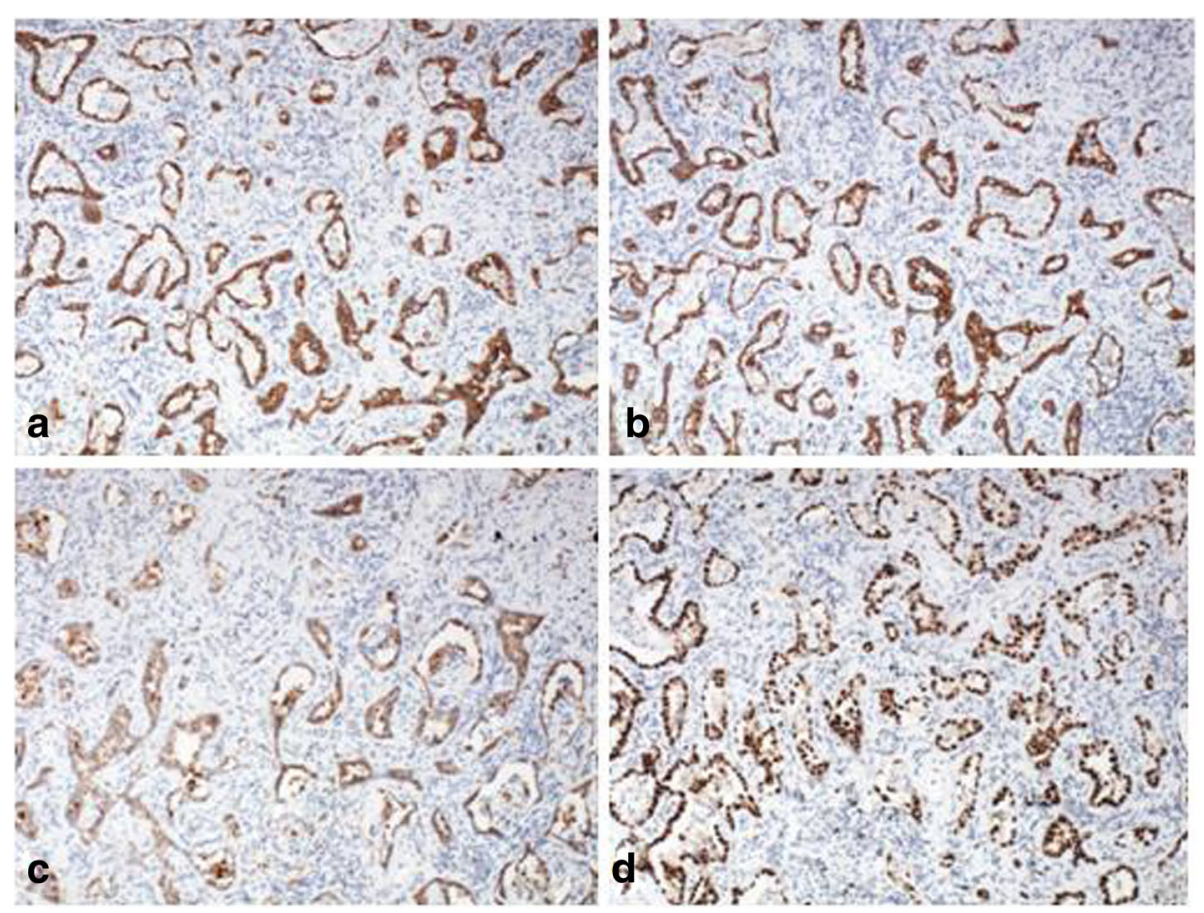

Fig. 6 Immunohistopathological analysis of the postoperative right lung lesion demonstrated positive staining for CK A1 (a), CK7 (b), napsin A (c), and TTF-1 (d) 
deletion in exon 19 (Fig. 7). The pathological TNM stage was pT1aNOM0, stage IA according to AJCC version 7.0 [5].

\section{Discussion and conclusions}

MPMT occur rarely, although consistent with the increased availability of diagnostic techniques, the number of patients diagnosed with MPMT is increasing. The diagnosis of MPLC can be difficult when multiple lung tumors of the same histologic type are present; similarly, it is not always easy to distinguish synchronous primary lesions from metastases. Analysis of gene mutations can be helpful in obtaining an accurate diagnosis. [6]

In the present case, the LLL lesion diagnosed concurrently with the breast cancer was also considered malignant, whereas the RLL lesion was not diagnosed as benign or malignant. We decided to surgically resect the breast cancer firstly. After that, we considered the LLL lesion was probably primary lung cancer instead of metastases from breast cancer because of the situ diagnosis in breast lesion. The pathological results of LLL showed that TTF-1 and Napsin A are positive and L858R mutation is detected in EGFR exon 21 which supported the diagnosis of primary lung cancer. To further identify the diagnosis of MPMT, we analyzed the EGFR mutation and TTF- 1 status in breast lesion and found they were both negative. TTF-1 has been wildly used for differential diagnosis from lung adenocarcinoma with others and can be a useful marker in identifying lung tissue as a primary origin of metastases [7-10]. These results confirmed the diagnosis of MPMT.

The pathological stage of breast cancer and lung cancer suggested that there is no need for adjuvant therapy according to NCCN guideline. But, interestingly, the RLL lesion which firstly presented as small ground glass density nodules had been gradually increased in size in 6 months after initial diagnosis. This phenomenon highly indicated the malignant behavior. However, it was unclear whether the RLL lesion was a primary lung cancer or a metastasis from the breast or LLL lung cancers. Since the RLL lesion remained stable after administration of gefitinib for 40 days, and the patient's performance status was suitable for surgery, we resected the RLL lesion. Pathological analysis confirmed that both the LLL and RLL lesions were adenocarcinoma of the lung with similar morphological features but with different EGFR mutations. The clinical and radiological presentation of the RLL lesion was indicative of a ground-glass opacity, and the pathological analysis confirmed a non-invasive adenocarcinoma. The sensitivity of the tumor to gefitinib treatment was consistent with the presence of EGFR mutation. The RLL and LLL lesions were considered to be of distinct origin because they contained different EGFR mutations.
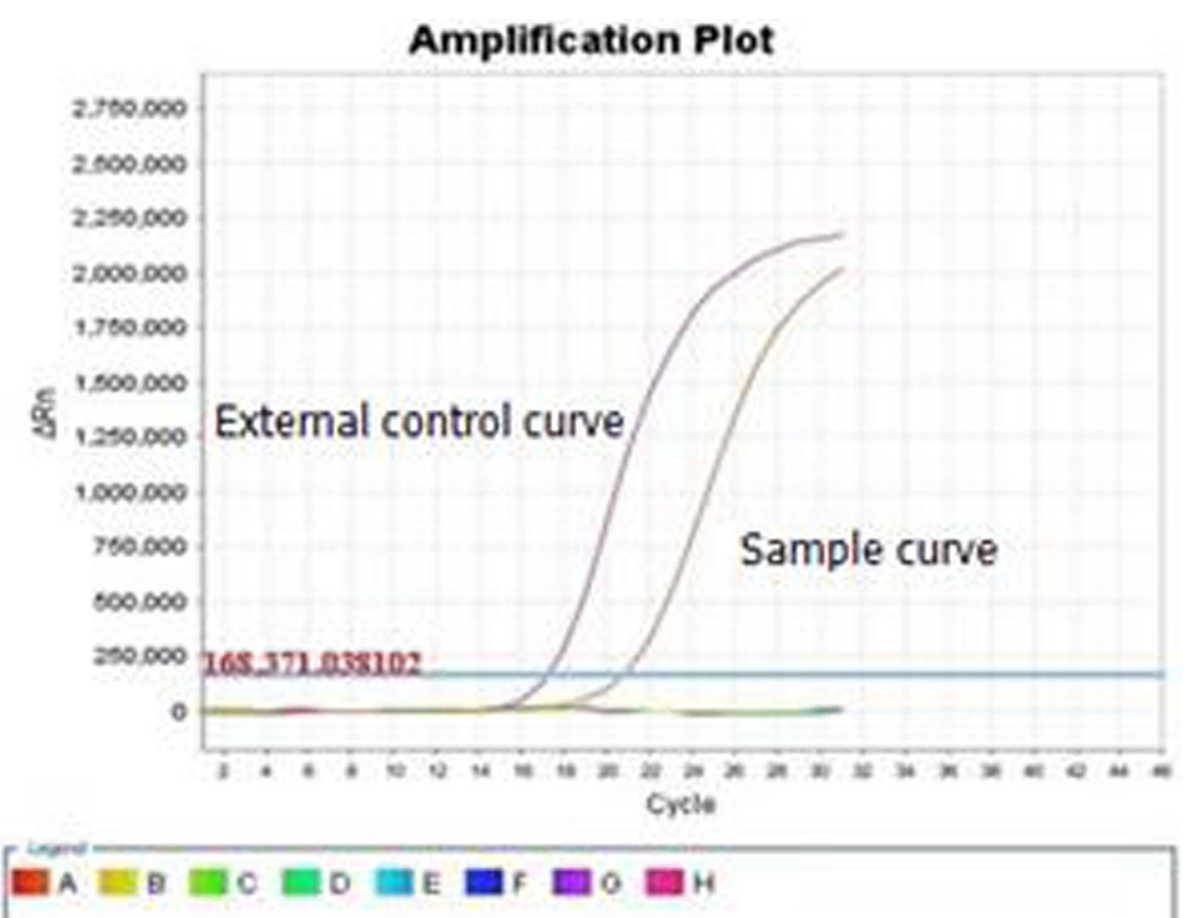

Fig. 7 EGFR gene analysis of the right lung lesion revealed a deletion in exon 19 
The final diagnosis in the present case was breast cancer with synchronous primary lung cancers. Given the different mutational status of the EGFR gene in the two lung adenocarcinomas, it is reasonable to assume that they are oncodevelopmentally independent. The patient remains disease-free as of Aug 16th 2018.

\section{Abbreviations}

EGFR: Epidermal growth factor receptor; ER: Estrogen receptor; FDG: $\mathrm{F}^{18}$-fluorodeoxyglucose; IHC: Immunohistochemical; LLL: Lower lung lobe; MPLC: Multiple primary lung cancer; MPMT: Multiple primary malignant tumors; NSCLC: Non small cell lung cancer; PET-CT: Positron emission tomography-computed tomography; PR: Progesterone receptor; RLL: Right lower lung lobe; SUV: Standardized uptake value; TNM: Tumor Lymph node Metastasis stage

\section{Acknowledge}

Not applicable

\section{Funding}

Our study protocol has undergone peer-review in writing the manuscript by the funding body (Planning Commission of science and Research Fund of Liaoning Province: Award Number: AFSOP-07-1.0-01).

\section{Availability of data and materials}

All data generated or analysed during this study are included in this published article.

\section{Authors' contributions}

$B J, X Q$ and $Y L$ were the treating physician of the patient. $B J, X Q, Y T$ and $Y L$ designed the study and drafted the manuscript. PY, YC and $C Z$ performed patient follow-up. XFC and YF analysed and interpreted the data. SZ and XC contributed to the critical revision of the manuscript. XL prepared the figures, and XSQ contributed to the clinical diagnosis. All authors read and approved the final manuscript.

\section{Ethics approval and consent to participate}

Not applicable.

\section{Consent for publication}

Written informed consent was obtained from the patient's next of kin for publication of this case report and accompanying images.

\section{Competing interests}

The authors declares that they have no competing interests.

\section{Publisher's Note}

Springer Nature remains neutral with regard to jurisdictional claims in published maps and institutional affiliations.

\section{Author details}

'Department of Medical Oncology, The First Hospital, China Medical University, Shenyang, China. ${ }^{2}$ Department of Pathology, The First Hospital, China Medical University, Shenyang, China. ${ }^{3}$ Department of pathology, Shengjing Hospital, China Medical University, Shenyang, China.

Received: 6 December 2017 Accepted: 30 October 2018

Published online: 20 November 2018

\section{References}

1. Martini N, Melamed MR. Multiple primary lung cancers. J Thorac Cardiovasc Surg. 1975;70(4):606-12.

2. Spratt JS, Hoag MG. Incidence of multiple primary cancers per man -year of followup: 20- year review from the Ellis Fischel state Cancer hospital. AnnSurg. 1966;164(5):775-84.

3. Aydiner A, Karadeniz A, Uygun K. Multiple primary neoplasms at a single institution: differences between synchronous and metachronous neoplasms. Am J Clin Oncol. 2000;23(4):364-70.
4. Edge $S B$, Byrd DR, Compton CC, et al. AJCC cancer staging manual[M]. New York: Springer; 2010. p. 347-76.

5. Rami-Porta R, et al. The IASLC lung Cancer staging project. J Thoracic Oncol. 2015:10:990-1003.

6. Zhu Z, Yu T, Chai Y. Multiple primary lung cancer displaying different EGFR and PTEN molecular profiles. Oncotarget. 2016;7(49):81969-71. https://doi. org/10.18632/oncotarget.13046

7. Ordonez NG. Thyroid transcription factor-1 is a marker of lung and thyroid carcinomas. Adv Anat Pathol. 2000;7(2):123-7. https://doi.org/10.1097/ 00125480-200007020-00007.

8. Puglisi $F$, et al. Prognostic value of thyroid transcription factor-1 in primary, resected, non-small cell lung .PubMed PMID: 10102618.carcinoma. Mod Pathol. 1999;12(3):318-24.

9. Di Loreto C, et al. TTF-1 protein expression in pleural malignant mesotheliomas adenocarcinomas of the lung. Cancer Lett. 1998:124(1):73-8 PubMed9500194.

10. Di Loreto C, et al. And PMID: Immunocytochemical expression of tissue specific transcription factor-1 in lung carcinoma. J Clin Pathol. 1997;50(1): 30-2 PubMed PMID: 9059352; PubMed Central PMCID: PMC499708.

\section{Ready to submit your research? Choose BMC and benefit from:}

- fast, convenient online submission

- thorough peer review by experienced researchers in your field

- rapid publication on acceptance

- support for research data, including large and complex data types

- gold Open Access which fosters wider collaboration and increased citations

- maximum visibility for your research: over $100 \mathrm{M}$ website views per year

At $\mathrm{BMC}$, research is always in progress.

Learn more biomedcentral.com/submissions 